Список использованной литературь:

1 Харасахал В.Х. Почти-периодические решения обыкновенных дифференциильных уравнений. Алма-Ата: Наука, Каз. ССР 1970. 200 с.

2 Умбетжанов Д.У. Почти многопериодические решения дифференциальных уравнений в частных производных. Алма-Ата: Наука, 1979. - 210 с.

3 Мухамбетова А.А., Сартабанов Ж.А. Устойчивость решений систем дифференциальных уравнений с многомерным временем. Актобе: Принт А, 2007. 168 с.

4 Кульжумиева А.А, Сартабанов Ж.А. Периодические решения систем дифференциильных уравнений с многомерным временем. Уральск: РИЦ ЗКГУ, 2013. $168 \mathrm{c.}$

5 Sartabanov Z.A. The multi-period solution of a linear system of equations with the operator of differentiation along the main diagonal of the space of independent variables and delayed arguments // AIP Conference Proceedings. - 2017. - Vol. 1880, P. 040020. https://doi.org/10.1063/1.5000636.

6 Ляпунов А.М. Общза задачи об устойчивости движений. М.-Л.: ГИТТЛ, 1950. - 472 с.

7 Малкин И.Г. Некоторые задачи теории нелинейных колебаний. М.: Гостехиздат, 1956. 492 c.

8 Сартабанов Ж.А., Омарова Б.Ж. Метод Ляпунова в исследовании многопериодических решений одной линейной системы уравнений с оператором дифференцирования // Таймановские чтения 2017: Труды международной научно-практической конференции, Уральск, Казахстан, 2017. С. 67-72.

9 Sartabanov Zh.A., Omarova B.Zh. Multiperiodic solutions of the one autonomous system of equations with the operator of differentiation with respect to spatial and time variables // Scientific journal Vestnik of Aktobe's K.Zhubanov Regional State University. 2018. - №1(51). - P.60-64.

10 Sartabanov, Z.A., Omarova, B.Z. Multiperiodic solutions of autonomous systems with operator of differentiation on the Lyapunov's vector field // AIP Conference Proceedings. 2018. Vol. 1997, P. 020041. https://doi.org/10.1063/1.5049035

11 Sartabanov Zh.A., Omarova B.Zh. On multi-periodic solutions of quasilinear autonomous systems with operator of differentiation on the Lyapunov's vector field // BULLETIN OF THE KARAGANDA UNIVERSITY-MATHEMATICS. 2019. - Vol. 94. №2. - P. 70-83. https://doi.org/10.31489/2019M2/70-83

12 Sartabanov Zh.A., Omarova B.Zh., Kerimbekov A. Research of multiperiodic solutions of perturbed linear autonomous systems with differentiation operator on the vector field // News of the National Academy of Sciences of the Republic of Kazakhstan. Physico-Mathematical Series. - 2019. Vol.6. №328. $\quad$ - P. $\quad 63-79$. https://doi.org/10.32014/2019.2518-1726.7

МРНТИ 27.39.15

УДК 517.5

DOI: https://doi.org/10.51889/2020-1.1728-7901.27

\title{
THE CONVOLUTION IN ANISOTROPIC TRIEBEL-LIZORKIN SPACES
}

\author{
Tleukhanova N.T. ${ }^{1}$, Sadykova K.K. ${ }^{1}$ \\ ${ }^{1}$ L.N. Gumilyov Eurasian National University, Nur-Sultan, Kazakhstan
}

\section{Abstract}

In this paper, we investigate the boundedness of the norm of the convolution operator in anisotropic Triebel-Lizorkin spaces. The Triebel-Lizorkin spaces are based on the Lorentz spaces $L_{p q}$. In the anisotropic case, we take the anisotropic Lorentz space $L_{\mathrm{pq}}$ as the base. The main goal of the paper is to solve the following problem: let $f$ and $g$ be functions from some classes of the Triebel-Lizorkin space scale. It is necessary to determine which conditions on the parameters of the spaces from $f$ and $g$ are taken and study which space belongs to their convolution $f * g$. An analogue of the O'Neil theorem was obtained for the Triebel-Lizorkin space scale $F_{\mathbf{p} \boldsymbol{\alpha}}^{\boldsymbol{\alpha q}}$, where $\boldsymbol{\alpha}, \boldsymbol{\tau}, \mathbf{p}, \mathbf{q}$ are vector parameters. Relations of the form $F_{\mathbf{r} \boldsymbol{\mu}}^{\beta \boldsymbol{\eta}} * F_{\mathbf{h v}}^{\gamma \xi} \hookrightarrow F_{\mathbf{p} \tau}^{\alpha \mathbf{q}}$ are obtained, with the corresponding ratios of vector parameters $\boldsymbol{\alpha}=\boldsymbol{\beta}+\boldsymbol{\gamma}, 1+\frac{1}{\mathbf{p}}=\frac{1}{\mathbf{r}}+\frac{1}{\mathbf{h}}, \frac{1}{\boldsymbol{\tau}}=\frac{1}{\boldsymbol{\mu}}+\frac{1}{\mathbf{v}}, \frac{1}{\mathbf{q}}=\frac{1}{\xi}+\frac{1}{\boldsymbol{\eta}}$. The research method is the functional spaces theory and inequalities of functional and harmonic analysis.

Keywords: Young-O’Neil inequality, anisotropic Triebel-Lizorkin spases, convolution. 


\title{
Аңุдатпа \\ Н.Т.Тлеуханова ${ }^{l}$, К.К.Садькова ${ }^{1}$ \\ ${ }^{1}$ Л.Н. Гумилев атындавы Еуразия ұлттық университеті, Нұр-Сұлтан қ., Қазақстан АНИЗОТРОПТЫ ТРИБЕЛЬ-ЛИЗОРКИН КЕНІСТІКТЕРІНДЕГІ ҮЙІРТКІ
}

Берілген жұмыста үйірткі операторы нормасының анизотропты Трибель-Лизоркин кеңістіктеріндегі шенелуі зерттеледі. Трибель-Лизоркин кеңістіктері $L_{p q}$ Лоренц кеңістіктерінің негізінде құрылады. Анизотропты жағдайда база ретінде анизотропты $L_{\text {pq }}$ Лоренц кеңістігін аламыз. Жұмыстың негізгі мақсаты келесі есепті шешу болып табылады: айталық, $f$ және $g$ Трибель-Лизоркин кеңістігі шкаласының қандай да бір класынан болсын. $f$ және $g$ алынатын кеңістіктің параметрлеріне қойылатын шарттарды анықтау және осы функциялардын $f * g$ үйірткісі қай кеңістікке жататынын анықтау қажет. Жұмыста $F_{\mathbf{p} \tau}^{\mathbf{\alpha q}}$ Трибель-Лизоркин кеңістігінің шкаласы үшін О’Нейл теоремасының аналогы алынды, мұнда $\boldsymbol{\alpha}, \boldsymbol{\tau}, \mathbf{p}, \mathbf{q}$ векторлық параметрлер болып табылады. $F_{\mathbf{r \mu}}^{\boldsymbol{\beta \eta}} * F_{\mathbf{h} \boldsymbol{v}}^{\gamma \xi} F_{\mathbf{p} \boldsymbol{\tau}}^{\boldsymbol{\alpha q}}$ түріндегі қатынас дәлелденді, мұнда қатынас параметрлеріне $\boldsymbol{\alpha}=\boldsymbol{\beta}+\boldsymbol{\gamma}, 1+\frac{1}{\mathbf{p}}=\frac{1}{\mathbf{r}}+\frac{1}{\mathbf{h}}$, $\frac{1}{\boldsymbol{\tau}}=\frac{1}{\boldsymbol{\mu}}+\frac{1}{\mathbf{v}}, \frac{1}{\mathbf{q}}=\frac{1}{\xi}+\frac{1}{\boldsymbol{\eta}}$ сәйкесінше шарттар қойылған. Зерттеу әдісі функционалдық анализдің теориясының тәсілдері мен функционалдық және гармоникалық анализ теңсіздіктерін қолдану болып табылады.

Түйін сөздер: Юнг-О’Нейл теңсіздігі, анизотропты Трибель-Лизоркин кеңістіктері, үйірткі.

\author{
Аннотация \\ Н.Т. Тлеуханова ${ }^{1}$, К.К. Садыкова ${ }^{1}$ \\ ${ }^{1}$ Евразийский национальный университет им. Л.Н. Гумилева, г. Нур-Султан, Казахстан \\ СВЕРТКА В АНИЗОТРОПНЫХ ПРОСТРАНСТВАХ ТРИБЕЛЯ-ЛИЗОРКИНА
}

В настоящей работе исследуется ограниченность нормы оператора свертки в анизотропных пространствах Трибеля-Лизоркина. Пространства Трибеля-Лизоркина базируются на основе пространств Лоренца $L_{p q}$. В анизотропном случае в качестве базы берем анизотропное пространство Лоренца $L_{\mathrm{pq}}$. Основной целью работы является решение следующей задачи: пусть $f$ и $g$ функции из некоторых классов шкалы пространств ТрибеляЛизоркина. Нужно определить условия на параметры пространств из которых берутся $f$ и $g$ и изучить класс, которому принадлежит их свертка $f * g$. В работе получен аналог теоремы О’Нейла для шкалы пространств Трибеля-Лизоркина $F_{\mathbf{p} \tau}^{\boldsymbol{\alpha q}}$, где $\boldsymbol{\alpha}, \boldsymbol{\tau}, \mathbf{p}, \mathbf{q}$ являются векторными параметрами. Получены соотношения вида $F_{\mathbf{r} \mu}^{\boldsymbol{\beta \eta}} * F_{\mathbf{h v}}^{\gamma \xi} \hookrightarrow F_{\mathbf{p} \tau}^{\mathbf{\alpha q}}$, при соответствующих соотношениях векторных параметров $\boldsymbol{\alpha}=\boldsymbol{\beta}+\boldsymbol{\gamma}, 1+\frac{1}{\mathbf{p}}=\frac{1}{\mathbf{r}}+\frac{1}{\mathbf{h}}$, $\frac{1}{\boldsymbol{\tau}}=\frac{1}{\boldsymbol{\mu}}+\frac{1}{\mathbf{v}}, \frac{1}{\mathbf{q}}=\frac{1}{\xi}+\frac{1}{\boldsymbol{\eta}}$. Методом исследования являются аппараты теории функциональных пространств и неравенства функционального и гармонического анализа.

Ключевые слова: неравенства Юнга-О’Нейла, анизотропные пространства Трибеля-Лизоркина, свертка.

\section{Introduction}

Let $I$ be either a $n$-dimensional torus $\mathbf{T}^{n}=[0,1)^{n}$, or a Euclidean space $\mathbf{R}^{n}$. Let $f(x)$ and $g(x)$ be determined and measurable functions on $I$ with respect to the $n$-dimensional Lebesgue measure such that for almost all $x \in I$ there exists an integral

$$
\int_{I} f(x-y) g(y) d y .
$$

In this case, it is said that the convolution of these functions is defined

$$
(f * g)(x)=\int_{I} f(x-y) g(y) d y
$$

The classical Young's inequality [1] has the form: suppose 


$$
1 \leq p, r, q \leq \infty, \quad \frac{1}{q}+1=\frac{1}{p}+\frac{1}{r} .
$$

If $f \in L_{p}(I), g \in L_{r}(I)$, then almost everywhere in $I$ there exists a convolution $f * g$, belonging to the space $L_{q}(I)$ and the following inequality holds

$$
\|f * g\|_{L_{q}(I)} \leq\|f\|_{L_{p}(I)}\|g\|_{L_{r}(I)} .
$$

We write this statement in the form of a relation

$$
L_{p}(I) * L_{r}(I) \hookrightarrow L_{q}(I) .
$$

These inequalities play an important role in harmonic analysis and in the theory of partial differential equations [1].

If

$$
1<p, r, q<\infty, \frac{1}{q}+1=\frac{1}{p}+\frac{1}{r},
$$

then for $g_{0}(x)=\frac{1}{|x|^{\frac{n}{r}}}$ the inequality holds

$$
\left\|f * g_{0}\right\|_{L_{q}(I)} \leq C\|f\|_{L_{p}(I)} .
$$

This inequality is called the Hardy-Littlewood-Sobolev inequality. It does not follow from Young's inequality, since $\left\|g_{0}\right\|_{L_{r}(I)}=\infty$. Generalization of inequality (1.3) obtained by O'Neil [2].

If (1.4) is true and $0<s_{1}, s_{2}, s \leq \infty, \frac{1}{s}=\frac{1}{s_{1}}+\frac{1}{s_{2}}$, then

$$
L_{p s s_{1}} * L_{r s_{2}} \hookrightarrow L_{q s}
$$

and in particular

$$
L_{p} * L_{r \infty} \hookrightarrow L_{q}
$$

where $L_{p s}$ is Lorentz space.

Note that in relation (1.5), condition (1.4) is essential. The limiting cases of the O'Neil inequality with condition (1.2) were considered in [3].

The O'Neil inequality for anisotropic Lorentz spaces was studied in [4,5]. In the case of $n \geq 2$ these results are extended the inequality (1.6). In the one-dimensional case, the O'Neil inequality was extended in [6,7].

There are generalizations of the Young and O'Neil inequalities for various functional spaces: weighted $L_{p}$ spaces, classical and Lorentz weighted spaces, Hardy spaces, Wiener spaces, Orlicz spaces; see [4], [8-11], and references therein.

Convolution operators were studied in spaces of smooth functions in [12-14].

In this paper, we investigate the boundedness of the convolution operator in anisotropic Triebel-Lizorkin spaces.

\section{Anisotropic Triebel-Lizorkin spaces}

Let $\mathbf{p}=\left(p_{1}, p_{2}\right), \boldsymbol{\tau}=\left(\tau_{1}, \tau_{2}\right), \boldsymbol{\mu}=\left(\mu_{1}, \mu_{2}\right), \mathbf{v}=\left(v_{1}, v_{2}\right), \mathbf{q}=\left(q_{1}, q_{2}\right), \boldsymbol{\alpha}=\left(\alpha_{1}, \alpha_{2}\right), \mathbf{k}=\left(k_{1}, k_{2}\right)$, $\mathbf{r}=\left(r_{1}, r_{2}\right), \mathbf{h}=\left(h_{1}, h_{2}\right), \boldsymbol{\beta}=\left(\beta_{1}, \beta_{2}\right), \boldsymbol{\gamma}=\left(\gamma_{1}, \gamma_{2}\right), \mathbf{x}=\left(x_{1}, x_{2}\right)$.

Let $1 \leq \mathbf{p}, \boldsymbol{\tau} \leq \infty$. For the function $f \in L_{\mathbf{p} \tau}\left(\mathbf{T}^{2}\right)$ let us define

$$
\Delta_{\mathbf{k}} f\left(x_{1}, x_{2}\right)=\sum_{m_{2}=2^{k_{2}}}^{2^{k_{2}+1}} \sum_{m_{1}=2^{k_{1}}}^{2^{k_{1}+1}} a_{\mathbf{m}}(f) e^{2 \pi i\left(m_{1} x_{1}+m_{2} x_{2}\right)},
$$

where the $\left\{a_{\mathbf{m}}(f): \mathbf{m} \in \mathbf{Z}^{2}\right\}$ are the Fourier coefficients of $f$ with respect to the multiple trigonometric system, $k \in \mathbf{N}$.

Let $-\infty<\boldsymbol{\alpha}<\infty$. 
The set of functions $f \in L_{\mathbf{p}, \boldsymbol{\tau}}\left(\mathbf{T}^{2}\right)$ for which the following norms

$$
\|f\|_{F_{\mathbf{p \tau}}^{\alpha \mathbf{q}}\left(\mathbf{T}^{2}\right)}=\left\|\left(\sum_{k_{2}=0}^{\infty}\left(\sum_{k_{1}=0}^{\infty}\left(2^{\alpha_{1} k_{1}+\alpha_{2} k_{2}}\left|\Delta_{\mathbf{k}} f\right|\right)^{q_{1}}\right)^{q_{2} / q_{1}}\right)^{1 / q_{2}}\right\|_{L_{\mathbf{p}, \tau}\left(\mathbf{T}^{2}\right)}
$$

are finite, are called as Triebel-Lizorkin type spaces $F_{\mathbf{p} \tau}^{\mathbf{\alpha q}}\left(\mathbf{T}^{2}\right)$. In the isotropic case, these spaces were investigated in [15], where the interpolation properties were studied.

We define the concept of convolution for the elements of these spaces.

Let $f=\sum_{k_{2}=-\infty}^{\infty} \sum_{k_{1}=-\infty}^{\infty} a_{k_{1}, k_{2}} e^{2 \pi i\left(k_{1} x_{1}+k_{2} x_{2}\right)}$ and $g=\sum_{k_{2}=-\infty}^{\infty} \sum_{k_{1}=-\infty}^{\infty} b_{k_{1}, k_{2}} e^{2 \pi i\left(k_{1} x_{1}+k_{2} x_{2}\right)}$ be trigonometric series. By convolution of these series we mean the series

$$
(f * g)\left(y_{1}, y_{2}\right)=\sum_{k_{2}=-\infty}^{\infty} \sum_{k_{1}=-\infty}^{\infty} a_{k_{1}, k_{2}}(f) b_{k_{1}, k_{2}}(g) e^{2 \pi i\left(k_{1} x_{1}+k_{2} x_{2}\right)} .
$$

\section{Proposition 2.1.}

$$
\Delta_{\mathbf{k}}(f * g)\left(y_{1}, y_{2}\right)=\int_{0}^{1} \int_{0}^{1} \Delta_{\mathbf{k}} f\left(x_{1}, x_{2}\right) \Delta_{\mathbf{k}} g\left(y_{1}-x_{1}, y_{2}-x_{2}\right) d x_{1} d x_{2} .
$$

Proof. We consider the following quantities

$$
\begin{gathered}
\Delta_{\mathbf{k}} f\left(x_{1}, x_{2}\right)=\sum_{m_{2}=2^{k_{2}}}^{2^{k_{2}+1}} \sum_{m_{1}=2^{k_{1}}}^{2^{k_{1}+1}} a_{m_{1}, m_{2}}(f) e^{2 \pi i\left(m_{1} x_{1}+m_{2} x_{2}\right)}, \\
\Delta_{\mathbf{k}} g\left(y_{1}-x_{1}, y_{2}-x_{2}\right)=\sum_{m_{2}=2^{k_{2}}}^{2_{m_{1}}^{k_{2}+1}} \sum_{2^{k_{1}}}^{2^{k_{1}+1}} b_{m_{1}, m_{2}}(g) e^{2 \pi i\left(m_{1}\left(y_{1}-x_{1}\right)+m_{2}\left(y_{2}-x_{2}\right)\right)} .
\end{gathered}
$$

According to Parseval's equality we have

$$
\begin{aligned}
\int_{0}^{1} \int_{0}^{1} \Delta_{\mathbf{k}} f\left(x_{1}, x_{2}\right) \Delta_{\mathbf{k}} g\left(y_{1}-x_{1}, y_{2}-x_{2}\right) d x_{1} d x_{2} \\
\quad=\int_{0}^{1} \int_{0}^{1} \sum_{m_{2}=2^{k_{2}}}^{2^{k_{2}+1}} \sum_{m_{1}=2^{k_{1}}}^{2^{k_{1}+1}} a_{m_{1}, m_{2}}(f) e^{2 \pi i\left(m_{1} x_{1}+m_{2} x_{2}\right)} \sum_{m_{2}=2^{k_{2}}}^{2^{k_{2}+1}} \sum_{m_{1}=2^{k_{1}}}^{2^{k_{1}+1}} b_{m_{1}, m_{2}}(g) e^{2 \pi i\left(m_{1}\left(y_{1}-x_{1}\right)+m_{2}\left(y_{2}-x_{2}\right)\right)} d x_{1} d x_{2} \\
=\sum_{m_{2}=2^{k_{2}}}^{2^{k_{2}+1}} \sum_{m_{1}=2^{k_{1}}}^{2^{k_{1}+1}} a_{m_{1}, m_{2}}(f) b_{m_{1}, m_{2}}(g) e^{2 \pi i\left(m_{1} y_{1}+m_{2} y_{2}\right)} .
\end{aligned}
$$

Theorem 2.1. Let $\boldsymbol{\alpha}, \boldsymbol{\beta}, \boldsymbol{\gamma} \in \mathbf{R}^{2}, \boldsymbol{\alpha}=\boldsymbol{\beta}+\boldsymbol{\gamma}, 1<\mathbf{p}, \mathbf{r}, \mathbf{h}<\infty, 1+\frac{1}{\mathbf{p}}=\frac{1}{\mathbf{r}}+\frac{1}{\mathbf{h}}, 1 \leq \boldsymbol{\tau}, \boldsymbol{\mu}, \mathbf{v}<\infty, \frac{1}{\boldsymbol{\tau}}=\frac{1}{\boldsymbol{\mu}}+\frac{1}{\mathbf{v}}$ $1 \leq \mathbf{q}, \boldsymbol{\xi}, \boldsymbol{\eta} \leq \infty, \frac{1}{\mathbf{q}}=\frac{1}{\xi}+\frac{1}{\boldsymbol{\eta}}$.

Suppose that $f$ and $g$ are respectively measurable on $[0,1]^{2}$ and $[-1,1]^{2}$ functions such that $f \in F_{\mathbf{r \mu}}^{\boldsymbol{\beta \eta}}\left([0,1]^{2}\right), g \in F_{\mathbf{h v}}^{\gamma \xi \xi}\left([0,1]^{2}\right)$ and

$$
\|f * g\|_{F_{\mathbf{p \tau}}^{\alpha \mathbf{q}}} \leq C\|f\|_{F_{\mathrm{r} \mu}^{\beta \mu}}\|g\|_{F_{\mathbf{h v}}^{\gamma_{\mathrm{s}}^{*}}} .
$$


Proof. Applying Proposition 2.1, we derive

$$
\begin{aligned}
\|f * g\|_{F_{\mathbf{p r}} \alpha_{\mathbf{q}}} & =\left\|\left(\sum_{k_{2}=0}^{\infty}\left(\sum_{k_{1}=0}^{\infty}\left(2^{\alpha_{1} k_{1}+\alpha_{2} k_{2}}\left|\Delta_{\mathbf{k}}(f * g)\right|\right)^{q_{1}}\right)^{q_{2} / q_{1}}\right)^{1 / q_{2}}\right\|_{L_{\mathbf{p r}}} \\
& =\left\|\left(\sum_{k_{2}=0}^{\infty}\left(\sum_{k_{1}=0}^{\infty}\left(2^{\alpha_{1} k_{1}+\alpha_{2} k_{2}}\left|\int_{0}^{1} \int_{0}^{1} \Delta_{\mathbf{k}} f(\mathbf{x}) \Delta_{\mathbf{k}} g(\mathbf{y}-\mathbf{x}) d x_{1} d x_{2}\right|\right)^{q_{1}}\right)^{q_{2} / q_{1}}\right)^{1 / q_{2}}\right\|_{L_{\mathbf{p r}}} \\
& \leq \| \int_{0}^{1} \int_{0}^{1}\left(\sum_{k_{2}=0}^{\infty}\left(\sum_{k_{1}=0}^{\infty}\left(2^{\alpha_{1} k_{1}+\alpha_{2} k_{2}}\left|\Delta_{\mathbf{k}} f(\mathbf{x}) \| \Delta_{\mathbf{k}} g(\mathbf{y}-\mathbf{x})\right|^{q_{1}}\right)^{q_{2} / q_{1}}\right)^{1 / q_{2}} d x_{1} d x_{2} \|_{L_{\mathbf{p r}}} .\right.
\end{aligned}
$$

Thus, using Hölder's inequality for $\frac{1}{\mathbf{q}}=\frac{1}{\xi}+\frac{1}{\boldsymbol{\eta}}$, we have

$$
\begin{aligned}
\|f * g\|_{F_{\mathbf{p r}}}^{\text {aq }} & \left.\leq \| \int_{0}^{1} \int_{0}^{1}\left(\sum_{k_{2}=0}^{\infty}\left(\sum_{k_{1}=0}^{\infty}\left(2^{\beta_{1} k_{1}+\beta_{2} k_{2}} \mid \Delta_{\mathbf{k}} f(\mathbf{x})\right)\right)^{\xi_{1}}\right)^{\xi_{2} / \xi_{1}}\right)^{1 / \xi_{2}} \\
& \times\left(\sum_{k_{2}=0}^{\infty}\left(\sum_{k_{1}=0}^{\infty}\left(2^{\gamma_{1} k_{1}+\gamma_{2} k_{2}}\left|\Delta_{\mathbf{k}} g\left(y_{1}-x_{1}, y_{2}-x_{2}\right)\right|\right)^{\eta_{1}}\right)^{\eta_{2} / \eta_{1}}\right)^{1 / \eta_{2}} d x_{1} d x_{2} \|_{L_{\mathbf{p} \tau}} .
\end{aligned}
$$

Let

$$
\begin{gathered}
F\left(x_{1}, x_{2}\right)=\left(\sum_{k_{2}=0}^{\infty}\left(\sum_{k_{1}=0}^{\infty}\left(2^{\beta_{1} k_{1}+\beta_{2} k_{2}}\left|\Delta_{\mathbf{k}} f\left(x_{1}, x_{2}\right)\right|\right)^{\xi_{1}}\right)^{\xi_{2} / \xi_{1}}\right)^{1 / \xi_{2}}, \\
G\left(y_{1}-x_{1}, y_{2}-x_{2}\right)=\left(\sum_{k_{2}=0}^{\infty}\left(\sum_{k_{1}=0}^{\infty}\left(2^{\gamma_{1} k_{1}+\gamma_{2} k_{2}}\left|\Delta_{\mathbf{k}} g\left(y_{1}-x_{1}, y_{2}-x_{2}\right)\right|\right)^{\eta_{1}}\right)^{\eta_{2} / \eta_{1}}\right)^{1 / \eta_{2}} .
\end{gathered}
$$

Further, applying the O'Neil inequality for anisotropic Lorentz spaces [5] with parameters $1+\frac{1}{\mathbf{p}}=\frac{1}{\mathbf{r}}+\frac{1}{\mathbf{h}}$, $\frac{1}{\boldsymbol{\tau}}=\frac{1}{\boldsymbol{\mu}}+\frac{1}{\mathbf{v}}$, we derive

Thus,

$$
\|f * g\|_{L_{\mathrm{pr}}^{\mathrm{aq}}} \leq\|F * G\|_{L_{\mathrm{pr}}} \leq C\|F\|_{L_{\mathrm{ru}}}\|G\|_{L_{\mathrm{hv}}} .
$$

$$
\begin{aligned}
& \|f * g\|_{F_{\mathrm{pr}}^{\text {aq }}} \leq C\left\|\left(\sum_{k_{2}=0}^{\infty}\left(\sum_{k_{1}=0}^{\infty}\left(2^{\beta_{1} k_{1}+\beta_{2} k_{2}} \mid \Delta_{\mathbf{k}} f\right)^{\xi_{1}}\right)^{\xi_{2} / \xi_{1}}\right)^{1 / \xi_{2}}\right\|\left\|_{L_{\mathrm{ru}}}\right\|\left(\sum_{k_{2}=0}^{\infty}\left(\sum_{k_{1}=0}^{\infty}\left(2^{\gamma_{1} k_{1}+\gamma_{2} k_{2}}\left|\Delta_{\mathbf{k}} g\right|\right)^{\eta_{1}}\right)^{\eta_{2} / \eta_{1}}\right)^{1 / \eta_{2}} \|_{L_{\mathrm{hv}}} \\
& =C\|f\|_{F_{\mathrm{Fn}}^{\beta_{n}}}\|g\|_{F_{\mathrm{hv}}^{\gamma \xi}} .
\end{aligned}
$$


References

1 Bennett C., Sharpley R. Interpolation of Operators // Pure and Applied Mathematics 129, Boston, MA, Academic Press, INC. - 1988. - 469 p.

2 O'Neil R. Convolution operators and L $(p, q)$ spaces // Duke Math. J. - 1963. -V. 30. - P. 129-142

3 Nursultanov E., Tikhonov S. Convolution inequalities in Lorentz spaces // J. Fourier Anal. Appl. - 2011. - V. 17. - P. 486-505.

4 Blozinski A.P. On a convolution theorem for L $(p, q)$ spaces. Trans. Amer. Math. Soc. 1972. V. 164. P. $255-265$.

5 Tleukhanova N.T., Sadykova K.K. O'Neil-type inequalities for convolutions in anisotropic Lorentz spaces // Eurasian Mathematical Journal, - 2019.- V. 10. № 3. - P. 68-83.

6 Nursultanov E., Tikhonov S., Tleukhanova N. Norm inequalities for convolution operators // C. R. Acad. Sci. Paris. - 2009. - V. I. № 347. - P. 1385-1388.

7 Nursultanov E., Tikhonov S., Tleukhanova N. Norm convolution inequalities in Lebesgue spaces // Rev. Mat. Iberoam. - 2018. - V. 34. № 2. - P. 811-838.

8 Heil C. An introduction to weighted Wiener amalgams. In Wavelets and their applications // Allied Publishers, New Delphi. - 2003. - P. 183-216.

9 Nursultanov E.D., Tleukhanova N.T. Multipliers of Multiple Fourier Series// Proc. Steklov Inst. Math. - 1999. T.227. - P. 231-236.

10 Kerman R., Sawyer E. Convolution algebras with weighted rearrangement-invariant norm // Studia Math. - 1994. - V. 108. № 2. - P. 103-126.

11 Nursultanov E., Tikhonov S. Weighted norm inequalities for convolution and Riesz potential // Potential Analysis. - 2015. - № 42. № 2. - P. 435-456.

12 Batyrov B. E., Burenkov V. I. Estimates for convolutions in Nikol'skii-Besov spaces // Dokl. Akad. Nauk. - 1993. - V. 330. № 1. - P. 9-11.

13 Bui H. Weighted Young's inequality and convolution theorems on weighted Besov spaces // Math. Nachr. - 1994. - V. 170. - P. 25-37.

14 Sadykova K.K., Tleukhanova N.T. Estimates of the norm of the convolution operator in anisotropic Besov spaces with the dominated mixed derivative // Bulletin of the Karaganda University-Mathematics, - 2019. - V. 95. № 3. - P. 51-59.

15 Bekmaganbetov K., Nursultanov E. Interpolation of Besov $B_{p \tau}^{\sigma q}$ and Lizorkin-Triebel $F_{p \tau}^{\sigma q}$ spaces // Analysis Mathematica, - 2009. - V. 35. - P. 169-188.

\author{
Б. Шаріп ${ }^{l}$, А.T. Есимова ${ }^{l}$ \\ ${ }^{1}$ Қазақ ұлттық құыздар педагогикалық университеті, Алматы к.., Қазақсттан

\section{СЫЗЫҚТЫ ДИФФЕРЕНЦИАЛДЫҚ ТЕҢДЕУ ҮШІН БАСТАПҚЫ СЕКІРІСТІ ШЕТТІК ЕСЕП ШЕШІМІН БАҒАЛАУ}

\begin{abstract}
Аң̧датпа
Жұмыста үшінші ретті тұрақты коэффициентті сингулярлы ұйытқыған сызықты дифференциалдық теңдеу үшін шеттік есеп қарастырылған. Бұл есепте кіші параметр дифференциалдық теңдеу мен $\mathrm{t}=0$ нүктесіндегі шекаралық шарт құрамындағы жоғарғы туындылардың алдына қойылған. Біртекті сингулярлы ұйытқыған дифференциалдық теңдеудің іргелі шешімдер жүйесі оған сәйкес сипаттаушы теңдеудің түбірлері үшін алынған асимптотикалық жіктеулер негізінде құрылды. Осы жүйе Коши функциясын, арнайы шекаралық функцияларды және Грин функциясын құруда қолданылады. Аталған функциялардың көмегімен сингулярлы ұйытқыған шеттік есеп шешімінің аналитикалық формуласы алынды және $\mathrm{t}=0$ нүктесінде бұл шешімнің нөлінші ретті бастапқы секіріске ие болатыны анықталды. Қарастырылған сингулярлы ұйытқыған шеттік есеп шешімінің осы есептен $\varepsilon=0$ жағдайында алынған сәйкес ұйытқымаған есеп шешіміне ұмтылатыны дәлелденді.
\end{abstract}

Түйін сөздер: ұйытқу, сингулярлық, асимптотикалық баға, бастапқы секіріс, Коши есебі, шекаралық есеп. 